\title{
EXPLORACIÓN DEL IMPACTO DE LAS HABILIDADES PARA LA VIDA SOBRE CONDUCTAS DE RIESGO EN ADOLESCENTES
}

\author{
Marco Antonio SANTANA-CAMPAS \\ https://orcid.org/0000-0002-6750-2713 \\ IRMA CORTÉS \\ https://orcid.org/0000-0003-0717-4516 \\ SILVIA DOMÍNGUEZ \\ https://orcid.org/0000-0003-3884-1350 \\ Centro Universitario del Sur de la Universidad de Guadalajara, Jalisco, México \\ Correo electrónico: mascampas@gmail.com
}

Recibido: 2 de abril del 2021 / Aceptado: 6 de mayo del 2021

doi: https://doi.org/10.26439/persona2021.n024(1).5312

\begin{abstract}
Resumen. La adolescencia es una etapa caracterizada por cambios importantes a nivel biológico, psicológico y social, en la que aumenta la vulnerabilidad a conductas de riesgo. Se realizó una investigación no experimental, con un diseño de investigación-acción con el objetivo de promover las habilidades para la vida ( $\mathrm{HpV}$ ) como factor protector de conductas de riesgo en adolescentes de la Preparatoria Regional de Sayula, Jalisco. Los resultados mostraron que la constante exposición a un contexto que normaliza las conductas de riesgo juega un papel importante en la percepción de los adolescentes, quienes, al considerar que las vías de solución están ausentes, desarrollan conductas autodestructivas. Sin embargo, las intervenciones experienciales de las $\mathrm{HpV}$ desde los principios del enfoque centrado en la persona pueden ser una alternativa para la promoción, formación y aprendizaje-vivencia de las $\mathrm{HpV}$ y con esto un cambio en la percepción y atribución de las conductas de riesgo, relaciones familiares, reconocimiento y manejo de las emociones. Por ello, es necesario realizar intervenciones participativas con enfoque en $\mathrm{HpV}$ de modo que faciliten un ajuste social positivo en jóvenes y adolescentes.
\end{abstract}

Palabras clave: habilidades para la vida / factores de riesgo / conductas de riesgo / enfoque centrado en la persona / adolescencia 


\section{EXPLORING THE IMPACT OF LIFE SKILLS ON RISK BEHAVIORS IN ADOLESCENTS}

Abstract. Adolescence is a stage characterized by important biological, psychological and social changes, with an increased vulnerability to risk behaviours. A non-experimental study was conducted, with an action-research design. The purpose was to promote Life Skills (LSH) as a protective factor against risk behaviours in adolescents of the Regional High School of Sayula, Jalisco. The results showed that constant exposure to a context that normalizes risk behaviours plays an important role in the perception of adolescents; as they consider that the solution pathways are absent, they show self-destructive behaviours. However, experiential interventions of LSH from the principles of the Person-Centered Approach can be an alternative for the promotion, formation and learning-living of LSH and with this a change in the perception and attribution of risk behaviours, family relationships, recognition and management of emotions. Therefore, it is necessary to carry out participatory interventions with a focus on LSH so that they facilitate a positive social adjustment in young people and adolescents.

Keywords: life skills / risk factors / risk behaviors / person-centered approach / adolescence 


\section{INTRODUCCIÓN}

La adolescencia es una etapa del desarrollo humano ubicada entre la infancia y la adultez que se caracteriza por cambios importantes a nivel biológico, psicológico y social (Torres, 2014). De acuerdo con la Organización Mundial de la Salud (OMS, 2018), las particularidades de esta fase de la vida pueden aumentar la vulnerabilidad de los adolescentes a conductas de riesgo que implican un peligro para el bienestar. La misma OMS mencionó que en la adolescencia y juventud las principales causas y conductas de riesgo son el suicidio, el consumo de drogas, los accidentes de tránsito, la salud mental, entre otros.

Los trabajos de investigación sobre las habilidades sociales, o también llamadas habilidades para la vida, se han incrementado en los últimos años, debido principalmente a la relación que tienen con la promoción del desarrollo psicológico y social saludable (Sinergia, 2015).

Algunas de las investigaciones, como la de Corona y Peralta (2011), resaltan que las principales causas de mortalidad que afectan a la adolescencia son las conductas de riesgo; estas acrecientan la prevalencia de consumo de sustancias, el inicio de una vida sexual activa en edades tempranas, suicidio y conductas delictivas tales como robo y violencia. Gutiérrez, García-Saisó, Espinosa de la Peña y Balandrán (2016) asocian estos resultados con una exposición continua a comportamientos antisociales en el contexto latinoamericano.

Dicho de otra forma, se ha señalado que la sociedad y cultura juegan un papel determinante en esta situación, pues se ha identificado a la adolescencia como una de las etapas más vulnerables a las influencias del entorno, construyendo la creencia de que cuentan con poca capacidad de juicio ante el riesgo. Sin embargo, no se ha considerado la percepción que ellos tienen como agentes protagonistas en la situación (Correa, Stella y Ortiz, 2018).

Del mismo modo, este proceder se ha asociado a un desajuste o incompetencia social que puede obstaculizar el desarrollo de habilidades para la vida; las que son consideradas por la OMS como la base para el desarrollo humano, principalmente en edades tempranas de la vida, pues facilitan el desarrollo emocional, intelectual y social saludable (Torres, 2014).

Por lo anterior, los autores antes mencionados recomiendan el desarrollo juvenil positivo para ampliar la conciencia de los adolescentes de sus propias fortalezas y disminuir las conductas de riesgo. En una investigación realizada con 5651 estudiantes de 17 años, García (2009) encontró que los participantes que tenían puntuaciones más altas (38 \%) en las áreas de planeación del futuro, autocontrol, manejo de enojo, solución de problemas y resistencia a la presión eran menos propensos al consumo de tabaco, 
por lo que resalta la importancia de desarrollar programas de prevención enfocados en estas habilidades.

Asimismo, Pérez de la Barrera (2013) manifiesta la necesidad de desarrollar programas de prevención de conductas sexuales de riesgo para adolescentes desde un enfoque de $\mathrm{HpV}$, debido a que en su investigación con 489 estudiantes de entre 14 y 20 años del estado de Morelos, México, comprobó que el inicio de la vida sexual promedio era a los 14 años, lo que facilitaba la práctica de comportamientos sexuales de riesgo. Además, un alto porcentaje de adolescentes utiliza el condón solo de manera ocasional o no lo hace, por lo que hay una evidente necesidad de brindar educación sexual a los adolescentes que los adiestre en las habilidades de comunicación asertiva, toma de decisiones, negociación y conciencia de las consecuencias.

Por otra parte, Cardozo (2012), con el objetivo de indagar cómo se presentan las habilidades para la vida en los adolescentes e identificar los factores predictores de la empatía, realiza una investigación con 124 adolescentes escolarizados de entre 13 a 18 años de edad. Como resultado, encontró una correlación positiva entre las habilidades que tienen las intervenciones dirigidas a fomentar las habilidades para la vida como para afrontar situaciones y el manejo de sí mismo, así como entre las habilidades interpersonales de conducta asertiva, empatía y comunicación con habilidades para la toma de decisiones. Finalmente, señala como factores predictores de empatía el autoconcepto social, académico y una baja conducta agresiva, confirmando el valor que tienen las intervenciones como instrumento de desarrollo en los jóvenes.

De manera similar, Choque-Larrauri y Chirinos-Cáceres (2009), en una investigación conformada por 284 estudiantes de Perú de entre 13 y 16 años, buscaron determinar la eficacia de un programa de habilidades para la vida, encontrando un incremento significativo en las habilidades de comunicación y asertividad, mientras que en autoestima y toma de decisiones no se mostraron diferencias. Según señalan, son más complejas y requieren mayor tiempo para su desarrollo, con análisis afectivo, emocional y cognitivo. Concluyen que el aprendizaje de habilidades para la vida favorece el desarrollo de los estudiantes; sin embargo, es necesario que se siga trabajando en la efectividad de las intervenciones.

Díaz-Alzate y Mejía-Zapata (2018), por el contrario, en su estudio efectuado en Medellín, Colombia, con adolescentes de 12 a 14 años de tres instituciones, realizan una crítica argumentando que el modelo de habilidades para la vida existente es insuficiente para la prevención de conductas como el consumo temprano de sustancias psicoactivas y que al analizar los relatos de los adolescentes que han participado en estas investigaciones se muestra una percepción de un modelo que se limita a la enseñanza de conceptos. Como resultado, las acciones de enseñanza por medio de talleres y otras estrategias utilizadas con estos grupos no han tenido el impacto esperado para el 
desarrollo de entornos protectores frente a contextos que ponen en riesgo a los adolescentes. Por lo que proponen como alternativa el modelo de Martha Nussbaum, que considera las realidades de los adolescentes y sus contextos, lo que permite construir un modelo que se adapte a las necesidades de la población a intervenir.

De manera semejante, Tenorio (2017) evalúa un programa de prevención universal de acoso entre pares identificando que los adolescentes carecen de habilidades para la vida y presentan puntajes altos en acoso entre pares; en los hombres observó un puntaje significativo en conductas agresivas, mientras que en las mujeres un mayor puntaje en el acoso virtual. Concluye que es necesario elaborar un programa preventivo dirigido a estos adolescentes y modificarlo a lo largo de la investigación, ya que necesita ser adaptado a las características de la población atendida.

Por otra parte, Ayala-Avalos (2017) buscó a través de un taller formativo explicar cómo vive el adolescente de entre 15 y 16 años su sexualidad y cuáles son las características periféricas que acompañan esta etapa. Como resultado destaca que es importante que los adolescentes identifiquen las conductas de riesgo y que las escuelas implementen talleres para extender conductas alternativas que les permitan tomar decisiones más congruentes con su bienestar. Además, confirmó que cuando los estudiantes reciben información, disminuye el riesgo de embarazo y enfermedades de transmisión sexual.

Santana-Campas, Ramos Santana, Arellano Montoya y Martínez Ibarra (2020) identificaron niveles bajos en habilidades de empatía, solución de conflictos, pensamiento crítico y creativo, deficiente manejo del estrés; asimismo, en cuanto a las diferencias por sexo, encontraron que "el conocimiento de sí mismo es más bajo para mujeres y alto para hombres, en empatía fue más alta en mujeres en comparación con los hombres, en el manejo del estrés los resultados fueron más bajos en mujeres que en los hombres" (p. 519), por lo que recomiendan realizar intervenciones con enfoque en $\mathrm{HpV}$, no solo como talleres formativos, sino que se apueste por las metodologías participativas experienciales.

Las habilidades para la vida las entenderemos como los comportamientos positivos y adaptables que permiten a los individuos lidiar eficazmente con las demandas y los retos de la vida cotidiana; además, comprenden un grupo de competencias psicosociales y destrezas interpersonales que pueden orientarse para transformar el entorno de manera que incide positivamente en la salud (Mangrulkar, Whitman y Posner, 2001).

Asimismo, Gutiérrez (2016) las define como comportamientos aprendidos que se usan para enfrentar situaciones problemáticas de la vida diaria, se adquieren por experiencia directa o entrenamiento y su expresión es mediatizada por los contextos en que se producen las normas sociales y las expectativas de la comunidad. 
Al respecto, Santana-Campas, Ramos Santana, Arellano Montoya y Molina del Río (2018) afirman que las habilidades para la vida son aquellas que ayudan a promover el bienestar, unos resultados de salud positivos y un desarrollo asertivo. Como complemento Choque-Larrauri (2009), en una investigación, cita el concepto que proporciona el Ministerio de Salud del Perú como el conjunto de habilidades que permiten a las personas actuar de manera competente en las situaciones de la vida cotidiana, favoreciendo comportamientos saludables en las esferas física, psicológica y social.

Otro concepto, también proporcionado por el Ministerio de Salud del Perú (2005), define estas habilidades como destrezas para conducirse de cierta manera, de acuerdo con la motivación individual y el campo de acción que tenga la persona dentro de sus posibilidades sociales y culturales.

Al hacer la revisión teórica identificamos que las habilidades sociales y las habilidades para la vida en algunos estudios pueden utilizarse como sinónimos, ya que ambas se refieren a tipos similares de competencias psicosociales y estrategias preventivas para las conductas de riesgo relacionadas con la salud (Sinergia, 2015); por lo que es importante considerar la existencia de otros conceptos referidos a esta área, como las habilidades sociales, asertividad, habilidades psicosociales y competencia social (Choque-Larrauri y Chirinos-Cáceres, 2009).

\section{Dimensiones de las habilidades para la vida}

La OMS (2018) clasifica las habilidades sociales en diez dimensiones, las cuales a continuación se definen (Melero, como se citó en Díaz, Rosero, Melo y Aponte, 2013):

1. Conocimiento de sí mismo: reconocimiento de nuestra personalidad, características, idiosincrasia, fortalezas, debilidades, aspiraciones, expectativas, entre otros.

2. Empatía: capacidad para ponerse en el lugar del otro y desde esa posición captar sus sentimientos.

3. Comunicación asertiva y efectiva: habilidad para expresarse de manera apropiada al contexto relacional y social en que se vive.

4. Relaciones interpersonales: competencia para interactuar positivamente con las demás personas.

5. Toma de decisiones: capacidad para construir racionalmente las relaciones cotidianas de nuestra vida.

6. Solución de problemas y conflictos: destreza para enfrentar constructivamente las exigencias de la vida cotidiana. 
7. Pensamiento creativo: utilización de los procesos de pensamiento para buscar respuestas innovadoras a los diversos desafíos vitales.

8. Pensamiento crítico: capacidad para analizar con objetividad experiencias e información sin asumir pasivamente criterios ajenos.

9. Manejo de sentimientos y emociones: reconocimiento y gestión positiva de nuestro mundo emocional.

10. Manejo de tensiones y estrés: capacidad para reconocer nuestras fuentes de tensión y actuar positivamente para su control.

A manera de conclusión, podemos observar que las intervenciones formativasexperienciales en el área de habilidades para la vida son necesarias para el desarrollo juvenil positivo. Dichas intervenciones deben ser diseñadas y ajustadas de acuerdo con el contexto de desarrollo de los jóvenes, pues la evidencia ha demostrado que los programas que consideran las necesidades emergentes del ambiente social tienden a ser más efectivos.

Por lo anterior, fue que se planteó como objetivo promover las habilidades para la vida $(\mathrm{HpV})$ como factor protector de conductas de riesgo en adolescentes de la Preparatoria Regional de Sayula, Jalisco.

\section{METODOLOGÍA}

\section{Tipo de investigación}

Se realizó una investigación no experimental, de corte cualitativo, con un diseño de investigación-acción (Hernández-Sampieri y Mendoza, 2018). Se buscó que, a través de un taller formativo-experiencial, los participantes reflexionaran y tomaran decisiones sobre conductas de riesgo y las habilidades para la vida como mecanismo protector.

\section{Participantes}

Los participantes del estudio fueron ocho estudiantes con edades de entre 15 a 17 años de la Escuela Preparatoria Regional de la Universidad de Guadalajara de Sayula, Jalisco, México.

\section{Instrumentos}

Grupo focal. Se utilizó para explorar los conocimientos, experiencias y percepciones de los participantes; así como comprender los fenómenos sociales y psicológicos desde la perspectiva de las personas involucradas en la situación, siguiendo las recomendaciones de Hamui-Sutton y Varela-Ruiz (2013) para su elaboración. 
Observación participante. Se utilizó como técnica de recolección de información, permitiendo la aprehensión de la realidad vivida por los participantes mediante una recogida de información sistematizada y, por tanto, una aproximación a las perspectivas que estos tienen sobre la problemática estudiada (Fagundes Campos, Alves y Ribeiro, 2013).

Diario de campo. Se utilizó para registrar todo aquello susceptible de ser interpretado cualitativamente ayudando a sistematizar la experiencia, reelaborar y consolidar el conocimiento teórico-práctico en cualquiera de los campos de acción (Londoño, Ramírez, Londoño, Fernández y Vélez, 2009).

\section{Diseño de intervención}

Consistió en siete sesiones en las que se abordaron los temas de fortalecimiento del autoconocimiento y empatía, identificación y expresión emocional asertiva, manejo de ansiedad, comunicación efectiva, negociación y resolución de conflictos, toma de decisiones asertivas y, finalmente, un cierre para la intervención con conclusiones de los participantes sobre lo aprendido.

Las sesiones tenían una duración de una hora y treinta minutos, estuvieron estructuradas en tres partes: actividad pretexto, desarrollo del contenido, conclusiones y reflexiones finales. Cada actividad pretexto incluía actividades de análisis afectivo, cognitivo y conductual, con el objetivo de facilitar el desarrollo de las habilidades para la vida y su aplicación a su contexto inmediato.

En cada una de las sesiones se buscó aplicar los principios y condiciones para el cambio desde el enfoque centrado en la persona; esta era una apuesta por una plena confianza en las capacidades de las personas, centrándose en el aquí y ahora (SilvaContreras, 2017). Destaca al individuo como un ser que de manera activa busca su desarrollo respetando la libertad que tiene para decidir el rumbo de su vida y con la convicción de que la persona posee los recursos para hacerlo (Ayala-Avalos, 2017).

Se consideraron las siguientes condiciones facilitadoras para la intervención (Rogers, 1985):

- Aceptación positiva incondicional: se considera que el cambio se logra cuando la persona acepta la autenticidad de sí mismo y quien lo acompaña, permitiéndose experimentar y expresar sin juicio sus sentimientos.

- Empatía: fundamental para lograr cambios constructivos, pues las personas entran en una interacción de aceptación que se centra en el proceso de cada individuo.

- Congruencia: el facilitador evita interponer fachadas ante el cliente y permitirse 
ser él mismo, reconocerse como ser humano con sentimientos y experiencias propias que faciliten la confianza.

Se ha comprobado que estos tres elementos pueden facilitar el cambio en los llamados grupos de encuentro, al propiciar experiencias de crecimiento personal y mejorar el funcionamiento interpersonal (Cloninger, 2003). En este sentido, desde la intervención se reafirma la capacidad de los participantes para solucionar sus propios problemas y dar respuesta a sus necesidades, enfatizando la creación de un clima de seguridad, confianza psicológica y patrones de comunicación basados en la apertura personal, el respeto y aceptación.

Habría que decir que el ECP no es una percepción individualista, sino una forma de entender cómo interaccionan las necesidades individuales y sociales para el desarrollo de la conciencia personal y comunitaria (Gómez, 1999).

\section{Estrategia de análisis}

Se utilizó el método de análisis de contenido con perspectiva de conjunto y fenomenológica en cuatro pasos: categorización, estructuración, contrastación y teorización, esto desde la propuesta de Martínez (2004).

\section{Consideraciones éticas}

La participación de los adolescentes fue completamente voluntaria, los tutores firmaron el consentimiento informado y los adolescentes el asentimiento informado. Para garantizar la confidencialidad se cambiaron los nombres reales por seudónimos. Durante todo el proceso seguimos los principios de respeto, dignidad, cuidado responsable y responsabilidad social del código de ética del psicólogo mexicano. Además del consentimiento y asentimiento, las autoridades de la Preparatoria Regional de Sayula, Jalisco, aprobaron y dieron las facilidades para que la intervención se realizara en sus instalaciones.

\section{RESULTADOS Y DISCUSIÓN}

El objetivo general de esta investigación-intervención fue promover las habilidades para la vida como factor protector de conductas de riesgo en adolescentes de la Preparatoria Regional de Sayula, Jalisco. Se observó que los participantes lograron mejorar en las dimensiones de autoconocimiento, comunicación asertiva y efectiva, manejo de emociones y sentimientos, relaciones interpersonales, solución de problemas y conflictos, y pensamiento crítico.

Respecto a la percepción de los sujetos de estudio sobre los factores y conductas de riesgo al inicio de la intervención se encontró que estaba caracterizada por vulnerabilidad, pues en su discurso hablaban del consumo de sustancias como una alternativa 
a las dificultades que enfrentaban, preocupados por no ser escuchados por su familia o no encajar en los grupos de referencia y estándares sociales. Estas observaciones se relacionan con la idea de Beltrón (2012) de que los adolescentes buscan responder a la pregunta ¿vale la pena vivir o no? apoyándose en su entorno próximo y utilizando las conductas de riesgo como formas ambivalentes de expresar sus dificultades.

Lo anterior se infiere del discurso de un participante durante la intervención, mismo que mencionó:

Eso (el consumo de drogas) es una alternativa para dejar de pensar en la presión social y familiar [...] hay partes donde tienes que hacer esto para formar parte del barrio, incluso los llegan a obligar, para sentir pertenencia. [...] Vida solo hay una, de todas formas nos vamos a morir. (Ea-A)

Del mismo modo, Mietzel (2005) añade que los adolescentes y jóvenes ponen a prueba la autoridad intensificando los conflictos con los padres y propiciando un sentimiento de invulnerabilidad, lo que explicaría los errores de atribución causal presentados en el discurso de los participantes; por ejemplo, uno de ellos refirió: "Nos llama la atención lo prohibido... porque si no lo tomas, no estás de moda, es válido (el consumo de drogas) si no lo haces de manera constante... no estás dañando a nadie" (Cj-E). De este se infiere la necesidad de aceptación por medio de las conductas de riesgo y el error de atribución sobre las consecuencias del consumo de "drogas" en función de los otros, y no el riesgo personal.

Por otra parte, se resalta el papel de las redes sociales como un elemento de información para el aprendizaje del consumo de drogas: "Como en redes que subes video y se hace viral y ahí te dicen cómo consumir... son retos de internet para que consumas y, pues, lo haces" $(\mathrm{MgC})$, de esto se resalta la importancia del control parental para el uso y contenido de las redes.

Por lo anterior podemos asumir que los participantes manifestaban un discurso centrado en la prohibición, sin hacer hincapié en las consecuencias de sus decisiones, otorgando a sus conductas un sentido particular de pertenencia y búsqueda de identidad. Dicho de otro modo, existe una representación social respecto a las conductas de riesgo que se construye en la interacción que los individuos tienen con su contexto; de ahí que tanto los participantes como sus grupos de referencia otorguen un valor a dichos comportamientos, reforzados por los medios de comunicación, para, posteriormente, anclarse a su realidad como una conducta normal, es decir, funge como una forma de comprender e interpretar la realidad, que guía y orienta su conducta (Slapak y Grigoravicius, 2007).

Por el contrario, en la percepción del grupo sobre los factores y conductas de riesgo después de la intervención, manifestaron ser capaces de lidiar más eficazmente con las demandas y retos de la vida cotidiana. Además, disminuyó el error de atribución 
causal, aumentando el poder que se daban ellos mismos y su capacidad para dirigirse y controlarse. Aunque admitían que el contexto social tenía una fuerte influencia en sus conductas, ahora eran capaces de reconocer su propia responsabilidad al decidir en favor o en contra de dichas influencias sociales.

Los participantes de la intervención comenzaron a desarrollar sus destrezas psicosociales creando un clima de mayor seguridad y responsabilizándose de sí mismos; de acuerdo con Gutiérrez (2016), estas se adquieren por experiencia directa o entrenamiento y son expresadas en los contextos en que ellos se desenvuelven. También reportaron cambios respecto a la percepción que tenían del autoconocimiento, las emociones, el afrontamiento al estrés, la comunicación en sus familias y la forma en que tomaban decisiones. Al respecto un adolescente mencionó que "pues yo me acuerdo de que dije que no afectaba si consumes drogas una vez y que ya después no, pero ya ahora digo, es que sí, cuando las pruebas una vez, sí te afecta" (Ks-0, GF2-09/05/2019, 7), de esto se infiere que aumentó la percepción de riesgo en cuanto al consumo de drogas.

Otro participante, al final de la intervención, mencionó y reconoció la importancia de tomar decisiones tomando en cuenta sus consecuencias: "Considerar que, pues, cada decisión que tenemos va a tener consecuencias buenas, pero también consecuencias malas" (Ld-A, GF2-09/05/2019, 4); con esto podemos inferir que la intervención tuvo un efecto en la toma de decisiones con una visión de futuro en los participantes.

En cuanto a las $\mathrm{HpV}$ de manejo de estrés y emociones, cuando la promoción de estas se realiza desde una intervención vivencial y no solo formativa, se puede lograr una mayor conciencia sobre las emociones y la importancia de estas. Al respecto, dos adolescentes mencionaron que:

si no te conoces y no sabes manejar tu estrés, pues más fácil caes en esas cosas (refiriéndose a las drogas). Yo digo que por eso tienes que aplicar lo de la comunicación, hablar con tus padres o amigos y eso te va a ayudar más que irte a las drogas y quererte. (Ld-A, DCTC5-11/04/2019, 2)

$[\ldots]$

Yo solía pensar que las emociones no eran importantes y no me gustaba hablar de mis emociones, pero ahora sé que es bueno platicar sobre ello y ayuda a mejorar todo. (Kj-0, FE3-28/03/2019, 4)

El autoconocimiento y autoestima resultan ser elementos principales para la protección de conductas de riesgo y desarrollo de HpV. "Es importante conocernos a nosotros mismos... para poder tomar decisiones que nos favorezcan para nuestra vida... el beneficio también incluye a nuestro entorno, porque nos sentiremos a gusto con lo que somos" (Cj-E, FE1-14/03/2019,1); "Tienes que tener autoestima, quererte como eres y buscar gente que sí te quiere y te entienda y te escuche" (Cj-E, DCTC5-11/04/2019, 2). De esta manera, resulta pertinente promover estas $\mathrm{HpV}$ en los contextos escolares y familiares. 
En los grupos focales e instrumentos diagnósticos se detectó una deficiencia en la comunicación y en la percepción negativa de la relación con los padres; al final de la intervención, se rescata que al menos existió un cambio en la percepción de la relación familiar y la importancia de la comunicación y negociación: "Aprendí que puedo tener una comunicación asertiva con mis padres y así tener una mejor convivencia" ( $\mathrm{Mg}-\mathrm{C}$, FE4-04/04/2019, 3). "Aprendí que negociar puede generar beneficios a las personas o grupos... y puedo aplicarlo al pedir permiso con mis padres o para llegar a acuerdos con mis amigos" (El-R, FE6-02/05/2019, 3). Esto es un aprendizaje significativo desde la propia experiencia de los adolescentes que participaron en la intervención.

Los resultados de esta intervención coinciden con lo expuesto por Ayala-Avalos (2017) al señalar que contrario a lo que se cree de los jóvenes y adolescentes: estos tienen el potencial para desarrollarse y buscar su bienestar si se les proporcionan las condiciones adecuadas para desbloquear su tendencia a la autorrealización.

Además, Gutiérrez et al. (2016) mencionan que un factor determinante en el desarrollo pleno de las habilidades para la vida es la constante exposición que existe en el contexto de las conductas y factores nocivos; mismo que pudimos observar durante la intervención, ya que consideraban que la información que recibían en redes sociales y en su comunidad los incitaba a hacerlo para estar a la moda.

Por lo anterior, creemos que el contexto social juega un papel de suma importancia para que los jóvenes y adolescentes amplíen sus vías de solución; algunos antecedentes teóricos previamente ya citados como Corona y Peralta (2011) y Gutiérrez et al. (2016) refieren que en nuestra realidad cultural los factores y conductas de riesgo se han convertido en una forma de actuar normal, ya que, a través de las representaciones sociales que hemos construido, se les ha atribuido un significado de poder en el que se busca identidad y pertenencia; esto impacta en la percepción de los jóvenes que podrían evaluar como ausentes las vías de solución desencadenando conductas autodestructivas (Herrera, 1999).

Durante la intervención se observaron cambios favorables en la percepción del grupo, por lo que podemos señalar que es posible promover el uso de factores protectores en los jóvenes y adolescentes para construir una realidad social con un ajuste positivo. Sin embargo, la teoría de aprendizaje social, constructivista y de la conducta problemática establecen que para que estas conductas positivas se mantengan son esenciales las interacciones con el entorno, el tipo de retroalimentación que se recibe, así como las expectativas y valores que familias y amigos dan a estas nuevas conductas (Mangrulkar et al., 2001). Por tanto, se considera pertinente la constante exposición a factores protectores y ambiente positivo para el desarrollo integral de los adolescentes; no basta solo una intervención transversal, esta exposición tendría que ser longitudinal. 
En este sentido, coincidimos con Choque-Larrauri y Chiniros-Cáceres (2009) y DíazAlzate y Mejía-Zapata (2018) respecto a que se debe seguir trabajando en la efectividad de los programas de intervención de modo tal que faciliten e integren una estrategia mucho más completa, con un tiempo de exposición más prolongado y aplicable a otros contextos fuera de la intervención para generar un impacto significativo en los jóvenes y la comunidad.

\section{CONCLUSIONES}

El modelo de habilidades para la vida continúa siendo necesario para el desarrollo saludable de los adolescentes, pues las habilidades para la vida, como factores protectores, pueden disminuir la vulnerabilidad ante los factores y conductas de riesgo. Si además de lo anterior consideramos que en nuestro contexto los factores de riesgo son los que principalmente vulneran la salud de la población en general, al estar relacionados con problemas de alcoholismo, drogadicción, violencia, criminalidad y salud mental, adquiere mayor relevancia el seguir desarrollando estudios que amplíen el conocimiento en esta área de la investigación.

Las $\mathrm{HpV}$, sin duda, tienen un potencial para hacer frente a las situaciones cotidianas que pueden exponer a diferentes riesgos a los adolescentes, pero la promoción y formación de las $\mathrm{HpV}$ no han resultado una estrategia eficaz cuando solo se tiene el componente cognitivo. Resulta pertinente que se realicen intervenciones experienciales para que los participantes logren pensar, sentir y vivir el efecto positivo de las $\mathrm{HpV}$ en su contexto inmediato. Asimismo, se resalta la necesidad del abordaje interdisciplinario y la promoción experiencial de las $\mathrm{HpV}$.

Tal como señalan la teoría psicosocial y del comportamiento, no solo depende de características individuales, sino que responde a una serie de sistemas sociales y condiciones socioculturales, por lo que no considerar estas variables puede mermar el progreso de los adolescentes respecto a los factores protectores que los lleven a un ajuste social positivo. Para futuras investigaciones sugerimos que:

1. Las intervenciones amplíen su contexto de aplicación invitando a padres y a personas de la comunidad a que participen de la promoción experiencial de la HpV, ya que son ellos quienes dificultan o facilitan, en interacción con los sistemas sociales, orientan o retroalimentan la conducta.

2. El diseño de la intervención integre actividades que impacten en los tres niveles de análisis que requieren las habilidades para la vida: cognitivo, afectivo y conductual, ya que observamos que una de las debilidades de este estudio fue que las actividades estaban destinadas principalmente a fortalecer la capacidad de análisis, cognitiva y afectiva. 


\section{REFERENCIAS}

Ayala-Avalos, M. (2017). Sexualidad y conductas de riesgo en alumnos de bachillerato desde el enfoque centrado en la persona (tesis de maestría). ITESO, Jalisco, México. http://hdl.handle.net/11117/4947

Beltrón, D. (2012). Conductas de riesgo de los juegos de la muerte a los juegos del vivir. Topia Editorial.

Cardozo, G. (2012). Habilidades para la vida en adolescentes: factores predictores de la empatía. Anuario de Investigaciones de la Facultad de Psicología, 1(1), 83-93. https://revistas.unc.edu.ar/index.php/aifp/article/view/2900

Choque-Larrauri, R., y Chirinos-Cáceres, J. (2009). Eficacia del programa de habilidades para la vida en adolescentes escolares de Huancavelica, Perú. Revista de Salud Pública, 11(2), 169-181. https://doi.org/10.1590/S0124-00642009000200002

Cloninger, S. (2003). Teorías de la personalidad. Editorial Pearson Educación.

Corona, F., y Peralta, E. (2011). Prevención de conductas de riesgo. Revista Médica Clínica Las Condes, 22(1), 68-75. https://doi.org/10.1016/s0716-8640(11)70394-7

Correa, C., Stella, C. y Ortiz, M. (2018). Percepción del riesgo en la cotidianidad de los adolescentes. Revista Facultad Nacional de Salud Pública, 36(1). 45-54. https://doi. org/10.17533/udea.rfnsp.v36n1a06

Díaz, L., Rosero, R., Melo, M., y Aponte, D. (2013). Habilidades para la vida: análisis de las propiedades psicométricas de un test creado para su medición. Revista Colombiana de Ciencias Sociales, 4(2), 181-200. https://dialnet.unirioja.es/ descarga/articulo/5123783.pdf

Díaz-Alzate, M., y Mejía-Zapata, S. (2018). Desarrollo de habilidades para la vida en la prevención del consumo de sustancias psicoactivas: un enfoque crítico al modelo existente. Revista El Ágora USB, 18(1), 203-210. https://doi. org/10.21500/16578031.3450

Fagundes, V., Campos, A., Alves, C., y Ribeiro, C. (2013). Hablando de la observación participante en la investigación en el proceso salud mental-enfermedad. Index de Enfermería, 23(1-2), 75-79. http://www.index-f.com/index-enfermeria/v23n12/9228r.php

García, G. (2009). Habilidades para la vida y consumo de tabaco en adolescentes de bachillerato (tesis de posgrado). Universidad Autónoma de México. http://132.248.9.195/ ptd2009/octubre/0650832/Index.html

Gómez, J. (1999). Psicología de la comunidad; modelos y aplicaciones, un punto de vista humanista. Plaza \& Janés. 
Gutiérrez, J., García-Saisó, S., Espinosa-de la Peña, R., y Balandrán, D. (2016). Desigualdad en indicadores de comportamientos de riesgo en adolescentes en México: análisis de dos encuestas de salud. Salud Pública en México, 58(6), 657-665. https://doi.org/10.21149/spm.v58i6.7922

Gutiérrez, M. (2016). Habilidades para la vida: manual de conceptos básicos para facilitadores y educadores. Centro de Información y Educación para la Prevención del Abuso de Drogas. http://www.cedro.org.pe/emprendimientojuvenil/images/ pdf/habilidadesparalavidamanualdeconceptos16agosto.pdf

Hamui-Sutton, A., y Varela-Ruiz, M. (2013). La técnica de grupos focales. Investigación en Educación Médica, 2(5), 55-60. http://www.redalyc.org/articulo.oa?id=3497 33230009

Hernández-Sampieri, R., y Mendoza, C. (2018). Metodología de la investigación. Las rutas cuantitativa, cualitativa y mixta. McGraw-Hill Education.

Herrera, P. (1999). Principales factores de riesgo psicológicos y sociales en adolescentes. Revista Cubana de Pediatría, 71(1), 39-42. http://scielo.sld.cu/pdf/ped/ v71n1/ped06199.pdf

Londoño, L., Ramírez, L.A., Londoño, C. I., Fernández, S., y Vélez, E. (2009). Diario de campo y cuaderno clínico: herramientas de reflexión y construcción del quehacer del psicólogo en formación. Revista Electrónica de Psicología Social Poiésis, 17. https://doi.org/10.21501/16920945.195

Mangrulkar, L., Whitman, C. V., y Posner, M. (2001). Enfoque de habilidades para la vida para un desarrollo saludable de niños y adolescentes. Organización Panamericana de la salud. Fundación W.K. Kellogg. http://www.codajic.org/sites/www.codajic. org/files/Enfoque\%20de\%20Habilidades\%20para\%20la\%20vida\%200PS_0.pdf

Martínez, M. (2004). Ciencia y arte en la metodología cualitativa. Editorial Trillas.

Mietzel, G. (2005). Claves de la psicología evolutiva. Herder Editorial.

Ministerio de Salud del Perú. (2005). Orientaciones técnicas del tema de habilidades para la vida. Dirección General de Promoción de la Salud. ftp://ftp2.minsa.gob.pe/docconsulta/documentos/dgps/ORIENTACIONES\%20TECNICAS\%20EN\%20HPV.doc

Organización Mundial de la Salud. (2018). Adolescentes: riesgos para la salud y soluciones. https://www.who.int/es/news-room/factsheets/detail/adolescents-healthrisks-and-solutions

Pérez de la Barrera, C. (2013). Habilidades para la vida y uso de anticoncepción por tipo de pareja sexual en adolescentes. Enseñanza e Investigación en Psicología, 18(1), 35- 49. http://www.redalyc.org/articulo.oa?id=29228948003 
Rogers, C. (1985). El proceso de convertirse en persona. Paidós.

Santana-Campas, M., Ramos, C., Arellano, R., y Molina del Río, J. (2018). Propiedades psicométricas del test de habilidades para la vida en una muestra de jóvenes mexicanos. Avances en Psicología, 26(2), 225-232. https://doi.org/10.33539/ avpsicol.2018.v26n2.1193

Santana-Campas, M., Ramos, C., Arellano, R., y Martínez, J. (2020). Habilidades para la vida: alternativa para la formación integral en estudiantes universitarios. Revista Ciencias de la Educación, 30(56), 519-540. http://servicio.bc.uc.edu.ve/educacion/ revista/56/art01.pdf

Silva-Contreras, F. (2017). El enfoque centrado en la persona, una herramienta para la recuperación de la experiencia formativa para la inserción laboral y/o educativa de jóvenes (tesis de posgrado). ITESO, Jalisco, México. http://hdl.handle.net/11117/5206

Sinergia. (2015). Identificación del nivel de desarrollo de las habilidades sociales en adolescentes de la Ciudad de México. XXVI Congreso e investigación CUAMACMor. https://feriadelasciencias.unam.mx/anteriores/feria26/feria194_01_identificacion_del_nivel_de_desarrollo_de_las_habi.pdf

Slapak, S., y Grigoravicius, M. (2007). Consumo de drogas: la construcción de un problema social. Anuario de Psicología, 14, 239-249. http://www.redalyc.org/articulo.oa?id=369139943026

Tenorio, L. (2017). Habilidades para la vida: Programa para la prevención de la agresión entre pares en adolescentes de secundaria (tesis de posgrado). Universidad Nacional Autónoma de México. http://132.248.9.195/ptd2017/octubre/0766707/Index.html

Torres, M. (2014). Las habilidades sociales. Un programa de intervención en educación secundaria obligatoria (tesis de posgrado). Universidad de Granada, España. http://masteres.ugr.es/psicopedagogica/pages/info_academica/trabajo_fin_ de_master/tfmhabilidadessociales/ 